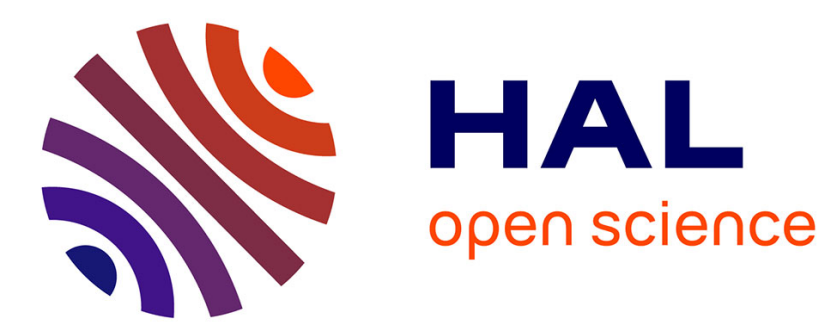

\title{
Capital-labor substitution and indeterminacy in continuous-time two-sector models
}

Jean-Philippe Garnier, Kazuo Nishimura, Alain Venditti

\section{To cite this version:}

Jean-Philippe Garnier, Kazuo Nishimura, Alain Venditti. Capital-labor substitution and indeterminacy in continuous-time two-sector models. 2006. halshs-00410713

\section{HAL Id: halshs-00410713 \\ https://shs.hal.science/halshs-00410713}

Preprint submitted on 24 Aug 2009

HAL is a multi-disciplinary open access archive for the deposit and dissemination of scientific research documents, whether they are published or not. The documents may come from teaching and research institutions in France or abroad, or from public or private research centers.
L'archive ouverte pluridisciplinaire HAL, est destinée au dépôt et à la diffusion de documents scientifiques de niveau recherche, publiés ou non, émanant des établissements d'enseignement et de recherche français ou étrangers, des laboratoires publics ou privés. 


\section{GREQAM}

Document de Travail

Groupement de Recherche en Economie $n^{\circ} 2006-35$ Quantitative d'Aix-Marseille - UMR-CNRS 6579 Ecole des Hautes Etudes en Sciences Sociales Universités d'Aix-Marseille II et III

Jean-Philippe GARNIER

Kazuo NISHIMURA

Alain VENDITTI

September 2006

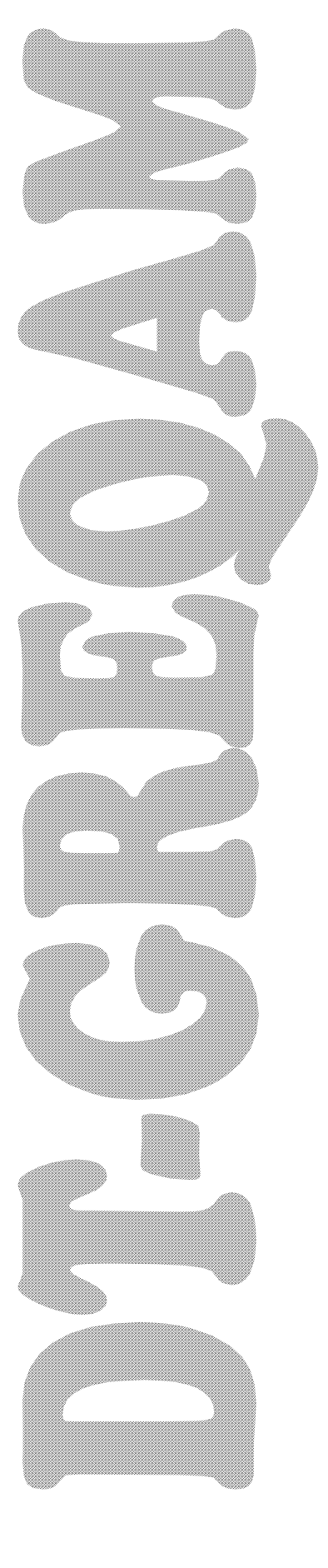




\title{
Capital-labor substitution and indeterminacy in continuous-time two-sector models*
}

\author{
Jean-Philippe GARNIER \\ Université de la Méditerranée, GREQAM, Marseille, France \\ Kazuo NISHIMURA \\ Institute of Economic Research, Kyoto University, Japan \\ and \\ Alain VENDITTI \\ CNRS - GREQAM, Marseille, France \\ First version: March 2005; Revised: August 2006
}

\begin{abstract}
The aim of this paper is to discuss the role of the elasticity of capital-labor substitution on the local determinacy properties of the steady state in a two-sector economy with CES technologies and sector-specific externalities.
\end{abstract}

Keywords: Sector-specific externalities, constant returns, capital-labor substitution, indeterminacy.

Journal of Economic Literature Classification Numbers: C62, E32, O41.

${ }^{*}$ We are grateful to T. Seegmuller and K. Shimomura for useful comments and suggestions. 


\section{Introduction}

Recently, a large number of papers have established the fact that locally indeterminate equilibria and sunspots fluctuations easily arise within twosector infinite-horizon growth models with sector specific external effects in production and linear preferences. ${ }^{1}$ For instance, considering Cobb-Douglas technologies, Benhabib and Nishimura [2] prove within a continuous-time model that the existence of local indeterminacy is obtained if and only if there is a reversal of factor intensities between the private and social levels. The consumption goods has indeed to be capital intensive from the private perspective but labor intensive from the social perspective.

When CES technologies are introduced, a much larger set of configurations is compatible with local indeterminacy. In particular, Leontief or linear technologies for the consumption good sector may be considered. Therefore, we will consider CES technologies in a continous-time model and provide a complete analysis of the local determinacy properties of equilibria. We will show that, even with asymmetric factor substitutability properties, local indeterminacy is still based on a reversal of factor intensities between the private and social levels, what we call quasi factor intensity. We will also show that local indeterminacy is compatible with a Leontief technology in the consumption good sector, and that this result is preserved if the elasticity of capital-labor substitution in that sector is not too large.

The paper is organized as follows: Section 2 presents the basic model with the production structure, the intertemporal equilibrium and the steady state. In Section 3 we provide all the results on the existence of local indeterminacy depending on different configurations for the CES coefficients at the private and social levels. Section 4 finally contains some concluding comments. All the proofs are gathered in a final Appendix.

\footnotetext{
${ }^{1}$ See Benhabib and Farmer [1].
} 


\section{The model}

\subsection{The production structure}

We consider an economy producing a pure consumption good $y_{0}$ and a pure capital good $y_{1}$. Each good is assumed to be produced by capital $x_{0 j}$ and labor $x_{1 j}, j=0,1$, through a CES technology which contains sector specific externalities. The representative firm in each industry indeed faces the following function:

$$
y_{j}=\left(\beta_{0 j} x_{0 j}^{-\rho_{j}}+\beta_{1 j} x_{1 j}^{-\rho_{j}}+e_{j}\left(X_{0 j}, X_{1 j}\right)\right)^{-1 / \rho_{j}}, \quad j=0,1
$$

with $\beta_{i j}>0, \rho_{j}>-1$ and $\sigma_{j}=1 /\left(1+\rho_{j}\right) \geq 0$ the elasticity of capital-labor substitution. The positive externalities are equal to

$$
e_{j}\left(X_{0 j}, X_{1 j}\right)=b_{0 j} X_{0 j}^{-\rho_{j}}+b_{1 j} X_{1 j}^{-\rho_{j}}
$$

with $b_{i j} \geq 0$ and $X_{i j}$ denoting the average use of input $i$ in sector $j$. We assume that these economy-wide averages are taken as given by each individual firm. At the equilibrium, since all firms of sector $j$ are identical, we have $X_{i j}=x_{i j}$ and we may define the social production functions as follows

$$
y_{j}=\left(\hat{\beta}_{0 j} x_{0 j}^{-\rho_{j}}+\hat{\beta}_{1 j} x_{1 j}^{-\rho_{j}}\right)^{-1 / \rho_{j}}
$$

with $\hat{\beta}_{i j}=\beta_{i j}+b_{i j}$. The returns to scale are therefore constant at the social level, and decreasing at the private level. We assume that in each sector $j=0,1, \hat{\beta}_{0 j}+\hat{\beta}_{1 j}=1$ so that the production functions collapse to Cobb-Douglas in the particular case $\rho_{j}=0$. Labor is normalized to one, i.e. $x_{00}+x_{01}=1$, and the total stock of capital is given by $x_{1}=x_{10}+x_{11}$.

Choosing the consumption good as the numeraire, i.e. $p_{0}=1$, a firm in each industry maximizes its profit given the output price $p_{1}$, the rental rate of capital $w_{1}$ and the wage rate $w_{0}$. Its profit is:

$$
\pi_{j}=p_{j} y_{j}-w_{0} x_{0 j}-w_{1} x_{1 j}
$$

The first order conditions subject to the private technologies (1) are

$$
p_{j} \beta_{i j}\left(y_{j} / x_{i j}\right)^{1+\rho_{j}}=w_{i}, \quad i, j=0,1
$$

From (3) we have

$$
x_{i j} / y_{j}=\left(p_{j} \beta_{i j} / w_{i}\right)^{\frac{1}{1+\rho_{j}}} \equiv a_{i j}\left(w_{i}, p_{j}\right), \quad i, j=0,1
$$


We call $a_{i j}$ the input coefficients from the private viewpoint. If the agents take account of externalities as endogenous variables in profit maximization, the first order conditions subject to the social technologies (2) are

$$
p_{j} \hat{\beta}_{i j}\left(y_{j} / x_{i j}\right)^{1+\rho_{j}}=w_{i}, \quad i, j=0,1
$$

and the input coefficients become

$$
\bar{a}_{i j}\left(w_{i}, p_{j}\right)=\left(p_{j} \hat{\beta}_{i j} / w_{i}\right)^{1 /\left(1+\rho_{j}\right)}, \quad i, j=0,1
$$

We call $\bar{a}_{i j}$ the input coefficients from the social viewpoint. We also define

$$
\hat{a}_{i j}\left(w_{i}, p_{j}\right) \equiv\left(\hat{\beta}_{i j} / \beta_{i j}\right) a_{i j}\left(w_{i}, p_{j}\right)
$$

as the quasi input coefficients from the social viewpoint, and it is easy to derive that

$$
\hat{a}_{i j}\left(w_{i}, p_{j}\right)=\bar{a}_{i j}\left(w_{i}, p_{j}\right)\left(\hat{\beta}_{i j} / \beta_{i j}\right)^{\rho_{j} /\left(1+\rho_{j}\right)}
$$

Notice that $\hat{a}_{i j}=\bar{a}_{i j}$ if there is no externality coming from input $i$ in sector $j$, i.e. $b_{i j}=0$, or if the production function is Cobb-Douglas, i.e. $\rho_{j}=0$. As we will show below, the factor-price frontier, which gives a relationship between input prices and output prices, is not expressed with the input coefficients from the social viewpoint but with quasi-input coefficients from the social viewpoint.

Based on these input coefficients straightforward computations allow to establish various Lemmas. We first show that the factor-price frontier is determined by the quasi input coefficients from the social viewpoint.

Lemma 1. Denote $p=\left(1, p_{1}\right)^{\prime}, w=\left(w_{0}, w_{1}\right)^{\prime}$ and $\hat{A}(w, p)=\left[\hat{a}_{i j}\left(w_{i}, p_{j}\right)\right]$. Then $p=\hat{A}^{\prime}(w, p) w$.

The factor market clearing equation depends on the input coefficients from the private perspective.

Lemma 2. Denote $x=\left(1, x_{1}\right)^{\prime}, y=\left(y_{0}, y_{1}\right)^{\prime}$ and $A(w, p)=\left[a_{i j}\left(w_{i}, p_{j}\right)\right]$. Then $A(w, p) y=x$.

Note that the rental rate is a function of the output price only, $w_{1}=$ $w_{1}\left(p_{1}\right)$, while outputs are functions of the capital stock and the output price, $y_{j}=y_{j}\left(x_{1}, p_{1}\right), j=0,1$.

We now examine some comparative statics. The factor-price frontier satisfies the Stolper-Samuelson theorem: 
Lemma 3. $d w_{1} / d p_{1}=\frac{\hat{a}_{00}}{\hat{a}_{11} \hat{a}_{00}-\hat{a}_{10} \hat{a}_{01}}$.

The factor market clearing equation finally satisfies the Rybczynski theorem:

Lemma 4. $d y_{1} / d x_{1}=\frac{a_{00}}{a_{11} a_{00}-a_{10} a_{01}}$.

Without external effects, i.e. $b_{i j}=0$, we have $\hat{A}(w, p)=A(w, p)$. The Rybczynski and Stolper-Samuelson theorems are equivalent since $[\partial y / \partial x]=$ $[\partial w / \partial p]$. However, in presence of externalities, the Rybczynski effects depend on the input coefficients from the private perspective while the StolperSamuelson effects depend on the quasi input coefficients from the social perspective. The duality between these two effects is thus destroyed. Local indeterminacy of equilibria will be a consequence of this property.

\subsection{Intertemporal equilibrium and steady state}

A representative agent optimizes a linear additively separable utility function with discount rate $\delta \geq 0$. This problem can be described as:

$$
\begin{aligned}
\max _{\left\{x_{i j}(t)\right\}} & \int_{0}^{+\infty}\left(\beta_{00} x_{00}(t)^{-\rho_{0}}+\beta_{10} x_{10}(t)^{-\rho_{0}}+e_{0}\left(X_{00}(t), X_{10}(t)\right)\right)^{-\frac{1}{\rho_{0}}} e^{-\delta t} d t \\
\text { s.t. } & y_{1}(t)=\left(\beta_{01} x_{01}(t)^{-\rho_{1}}+\beta_{11} x_{11}(t)^{-\rho_{1}}+e_{1}\left(X_{01}(t), X_{11}(t)\right)\right)^{-\frac{1}{\rho_{1}}} \\
& \dot{x}_{1}(t)=y_{1}(t)-g x_{1}(t) \\
& 1=x_{00}(t)+x_{01}(t) \\
& x_{1}(t)=x_{10}(t)+x_{11}(t) \\
& x_{1}(0) \text { and }\left\{e_{j}\left(X_{0 j}(t), X_{1 j}(t)\right)\right\}_{t \geq 0}, j=0,1, \text { given }
\end{aligned}
$$

where $g>0$ is the depreciation rate of the capital stock. We can write the modified Hamiltonian in current value as:

$$
\begin{aligned}
\mathcal{H} & =\left(\beta_{00} x_{00}(t)^{-\rho_{0}}+\beta_{10} x_{10}(t)^{-\rho_{0}}+e_{0}\left(X_{00}(t), X_{10}(t)\right)\right)^{-\frac{1}{\rho_{0}}} \\
& +w_{0}(t)\left(1-x_{00}(t)-x_{01}(t)\right)+w_{1}(t)\left(x_{1}(t)-x_{10}(t)-x_{11}(t)\right) \\
& +p_{1}(t)\left(\left(\beta_{01} x_{01}(t)^{-\rho_{1}}+\beta_{11} x_{11}(t)^{-\rho_{1}}+e_{1}\left(X_{01}(t), X_{11}(t)\right)\right)^{-\frac{1}{\rho_{1}}}-g x_{1}(t)\right)
\end{aligned}
$$


The static first order conditions are given by equations (3). The necessary conditions which describe the solution to the optimization problem are given by the following equations of motion:

$$
\begin{aligned}
& \dot{x}_{1}(t)=y_{1}\left(x_{1}(t), p_{1}(t)\right)-g x_{1}(t) \\
& \dot{p}_{1}(t)=(\delta+g) p_{1}(t)-w_{1}\left(p_{1}(t)\right)
\end{aligned}
$$

Any solution $\left\{x_{1}(t), p_{1}(t)\right\}_{t \geq 0}$ that also satisfies the transversality condition

$$
\lim _{t \rightarrow+\infty} e^{-\delta t} p_{1}(t) x_{1}(t)=0
$$

is called an equilibrium path.

A steady state is defined by a pair $\left(x_{1}^{*}, p_{1}^{*}\right)$ solution of

$$
\begin{aligned}
y_{1}\left(x_{1}, p_{1}\right) & =g x_{1} \\
w_{1}\left(p_{1}\right) & =(\delta+g) p_{1}
\end{aligned}
$$

We introduce the following restriction on parameters' values which will ensure the existence of an unique non trivial steady state:

Assumption 1. $\beta_{11}>\delta+g$ and $\rho_{1} \in\left(\hat{\rho}_{1},+\infty\right)$ with

$$
\hat{\rho}_{1} \equiv \frac{\ln \hat{\beta}_{11}}{\ln \left(\frac{\beta_{11}}{\delta+g}\right)-\ln \hat{\beta}_{11}} \in(-1,0)
$$

Considering the fact that, within continuous-time models, the discount rate $\delta$ and the capital depreciation rate $g$ are quite small, the restriction $\beta_{11}>\delta+g$ does not appear to be too demanding. Assumption 1 precisely guarantees positiveness and interiority of all the steady state values for input demand functions $x_{i j}$. Moreover it allows to prove the following result:

Proposition 1. Under Assumption 1, there exists a unique steady state $\left(x_{1}^{*}, p_{1}^{*}\right)>0$ such that

$$
\begin{aligned}
& x_{1}^{*}=\frac{\left(\frac{\beta_{10} \beta_{01}}{\beta_{00} \beta_{11}}\right)^{\frac{1}{1+\rho_{0}}}\left(\frac{\left(\frac{\beta_{11}}{\delta+g}\right)^{\frac{\rho_{1}}{1+\rho_{1}}}-\hat{\beta}_{11}}{\hat{\beta}_{01}}\right)^{\frac{1+\rho_{1}}{\rho_{1}\left(1+\rho_{0}\right)}}}{1-\left(\frac{\beta_{11}}{\delta+g}\right)^{\frac{1}{1+\rho_{1}}} g\left[1-\left(\frac{\beta_{10} \beta_{01}}{\beta_{00} \beta_{11}}\right)^{\left.\frac{1}{1+\rho_{0}}\left(\frac{\left(\frac{\beta_{11}}{\delta+g}\right)^{\frac{\rho_{1}}{1+\rho_{1}}}-\hat{\beta}_{11}}{\hat{\beta}_{01}}\right)^{\frac{\rho_{1}-\rho_{0}}{\rho_{1}\left(1+\rho_{0}\right)}}\right]}\right.} \\
& p_{1}^{*}=\frac{\beta_{10}}{\delta+g}\left[\hat{\beta}_{00}\left(\frac{\beta_{10} \beta_{01}}{\beta_{00} \beta_{11}}\right)^{\frac{\rho_{0}}{1+\rho_{0}}}\left(\frac{\left(\frac{\beta_{11}}{\delta+g}\right)^{\frac{\rho_{1}}{1+\rho_{1}}}-\hat{\beta}_{11}}{\hat{\beta}_{01}}\right)^{\frac{\rho_{0}\left(1+\rho_{1}\right)}{\rho_{1}\left(1+\rho_{0}\right)}}+\hat{\beta}_{10}\right]^{-\frac{1+\rho_{0}}{\rho_{0}}}
\end{aligned}
$$




\section{Main results}

We start by linearizing the dynamical system (7) around $\left(x_{1}^{*}, p_{1}^{*}\right)$ :

$$
J=\left(\begin{array}{cc}
\frac{\partial y_{1}}{\partial x_{1}}\left(x_{1}^{*}, p_{1}^{*}\right)-g & \frac{\partial y_{1}}{\partial p_{1}}\left(x_{1}^{*}, p_{1}^{*}\right) \\
0 & -\frac{\partial w_{1}}{\partial p_{1}}\left(p_{1}^{*}\right)+\delta+g
\end{array}\right)
$$

Any solution from (7) that converges to the steady state $\left(x_{1}^{*}, p_{1}^{*}\right)$ satisfies the transversality condition and is an equilibrium. Therefore, given $x_{1}(0)$, if there is more than one initial price $p_{1}(0)$ in the stable manifold of $\left(x_{1}^{*}, p_{1}^{*}\right)$, the equilibrium path from $x_{1}(0)$ will not be unique. In particular, if $J$ has two roots with negative real parts, there will be a continuum of converging paths and thus a continuum of equilibria.

Definition 1. If the locally stable manifold of the steady state $\left(x_{1}^{*}, p_{1}^{*}\right)$ is two-dimensional, then $\left(x_{1}^{*}, p_{1}^{*}\right)$ is said to be locally indeterminate.

The roots of $J$ are given by the diagonal terms. We know from Lemmas 3 and 4 that $\partial y_{1} / \partial x_{1}$ corresponds to the factor intensity difference from the private viewpoint and $\partial w_{1} / \partial p_{1}$ corresponds to the quasi factor intensity difference from the social viewpoint. Using the definitions of input coefficients given in Section 2, we may indeed interpret the elements of $\partial y_{1} / \partial x_{1}$ and $\partial w_{1} / \partial p_{1}$ as follows:

Definition 2. The consumption good is said to be:

i) capital intensive at the private level if and only if $a_{11} a_{00}-a_{10} a_{01}<0$,

ii) quasi capital intensive at the social level if and only if $\hat{a}_{11} \hat{a}_{00}-$ $\hat{a}_{10} \hat{a}_{01}<0$,

iii) capital intensive at the social level if and only if $\bar{a}_{11} \bar{a}_{00}-\bar{a}_{10} \bar{a}_{01}<0$.

We may thus relate the input coefficients to the CES parameters:

Proposition 2. Let Assumption 1 hold. At the steady state:

i) the consumption good is capital (labor) intensive from the private perspective if and only if 


$$
b \equiv 1-\left(\frac{\beta_{10} \beta_{01}}{\beta_{00} \beta_{11}}\right)^{\frac{1}{1+\rho_{0}}}\left(\frac{\left(\frac{\beta_{11}}{\delta+g}\right)^{\frac{\rho_{1}}{1+\rho_{1}}}-\hat{\beta}_{11}}{\hat{\beta}_{01}}\right)^{\frac{\rho_{1}-\rho_{0}}{\rho_{1}\left(1+\rho_{0}\right)}}<(>) 0
$$

ii) the consumption good is quasi capital (labor) intensive from the social perspective if and only if

$$
\hat{b} \equiv 1-\frac{\hat{\beta}_{10} \hat{\beta}_{01}}{\hat{\beta}_{00} \hat{\beta}_{11}}\left(\frac{\beta_{00} \beta_{11}}{\beta_{10} \beta_{01}}\right)^{\frac{\rho_{0}}{1+\rho_{0}}}\left(\frac{\left(\frac{\beta_{11}}{\delta+g}\right)^{\frac{\rho_{1}}{1+\rho_{1}}}-\hat{\beta}_{11}}{\hat{\beta}_{01}}\right)^{\frac{\rho_{1}-\rho_{0}}{\rho_{1}\left(1+\rho_{0}\right)}}<(>) 0
$$

iii) the consumption good is capital (labor) intensive from the social perspective if and only if

$$
\bar{b} \equiv 1-\left(\frac{\hat{\beta}_{10} \hat{\beta}_{01}}{\hat{\beta}_{00} \hat{\beta}_{11}}\right)^{\frac{1}{1+\rho_{0}}}\left(\frac{\left(\frac{\hat{\beta}_{11}}{\delta+g}\right)^{\frac{\rho_{1}}{1+\rho_{1}}}-\hat{\beta}_{11}}{\hat{\beta}_{01}}\right)^{\frac{\rho_{1}-\rho_{0}}{\rho_{1}\left(1+\rho_{0}\right)}}<(>) 0
$$

The following Proposition establishes that local indeterminacy requires a capital intensity reversal from the private input coefficients to the quasi input coefficients.

Proposition 3. Let Assumption 1 hold. The steady state is locally indeterminate if and only if the consumption good is capital intensive from the private perspective, but quasi labor intensive from the social perspective.

To get indeterminacy in a framework with constant returns to scale at the social level, we need a mechanism that nullify the duality between the Rybczynski and Stolper-Samuelson effects. As shown in Section 2.1, the Rybczynski effect is given by the input coefficients from the private perspective while the Stolper-Samuelson effect is given by the quasi input coefficients from the social perspective. In the presence of external effects, the duality between these coefficients is broken and local indeterminacy may appear.

This mechanism is very similar to the one exhibited in the contribution of Benhabib and Nishimura [2]. However in the current paper, it follows that depending on the value of the elasticities of capital-labor substitution, the capital intensity reversal from the private input coefficients to the quasi input coefficients does not necessarily requires a capital intensity reversal from the private to the social level.

Propositions 2 and 3 show that the stability properties of the steady state depend, among all the parameters, on whether the ratios $\beta_{10} \beta_{01} / \beta_{11} \beta_{00}$ and 
$\hat{\beta}_{10} \hat{\beta}_{01} / \hat{\beta}_{11} \hat{\beta}_{00}$ are lower or greater than 1 . Around the steady state, it can be easily shown that if the elasticities of capital-labor substitution are identical across sectors, the consumption good is capital intensive at the private level if and only if $\beta_{10} \beta_{01} / \beta_{11} \beta_{00}>1$ while it is capital intensive at the social level if and only if $\hat{\beta}_{10} \hat{\beta}_{01} / \hat{\beta}_{11} \hat{\beta}_{00}>1$. With asymmetric elasticities, as it is shown in Proposition 2, the capital intensity differences between sectors also depend on the parameters $\rho_{0}$ and $\rho_{1}$.

Notice also from Proposition 2 that the capital intensity differences at the private and quasi social level are linked as follows:

$$
\hat{b}=1-\frac{\frac{\hat{\beta}_{10} \hat{\beta}_{01}}{\hat{\beta}_{11} \hat{\beta}_{00}}}{\frac{\beta_{10} \beta_{01}}{\beta_{11} \beta_{00}}}(1-b)
$$

We know from Proposition 3 that local indeterminacy requires the consumption good to be capital intensive at the private level, i.e. $b<0$, but quasi labor intensive at the social level, i.e. $\hat{b}>0$. It follows from the above expression that when $b<0$, a necessary condition for $\hat{b}$ to be positive is given by the following Assumption:

Assumption 2. $\frac{\hat{\beta}_{10} \hat{\beta}_{01}}{\hat{\beta}_{11} \hat{\beta}_{00}}<\frac{\beta_{10} \beta_{01}}{\beta_{11} \beta_{00}}$

Let us first consider the configuration $\beta_{10} \beta_{01} / \beta_{11} \beta_{00}<1$ which is known in the case with symmetric elasticities of capital-labor substitution to imply local determinacy of the steady state. ${ }^{2}$ The following Proposition shows on the contrary that as soon as the elasticities are sufficiently asymmetric, there is room for local indeterminacy.

Proposition 4. Under Assumptions 1-2, let

$$
1>\frac{\beta_{10} \beta_{01}}{\beta_{11} \beta_{00}} \text { and } 1>\frac{\hat{\beta}_{10} \hat{\beta}_{01}}{\hat{\beta}_{11} \hat{\beta}_{00}}>\frac{\hat{\beta}_{01}}{\frac{\beta_{11}}{\delta+g}-\hat{\beta}_{11}}
$$

Then there are $\underline{\rho}_{0} \in(-1,0), \bar{\rho}_{0}>0$ such that the following results hold:

i) for any given $\rho_{0} \in\left(\underline{\rho}_{0}, \bar{\rho}_{0}\right)$, there exist $\underline{\rho}_{1}>\max \left\{0, \rho_{0}\right\}$ and $\bar{\rho}_{1}>\underline{\rho}_{1}$ such that the steady state is locally indeterminate when $\rho_{1} \in\left(\underline{\rho}_{1}, \bar{\rho}_{1}\right)$;

ii) for any given $\rho_{0}>\bar{\rho}_{0}$, there exists $\underline{\rho}_{1}>\rho_{0}$ such that the steady state is locally indeterminate when $\rho_{1}>\underline{\rho}_{1}$.

\footnotetext{
${ }^{2}$ See Benhabib and Nishimura [2] for Cobb-Douglas economies and Nishimura and Venditti [3] for CES economies with symmetric elasticities of capital-labor substitution.
} 
Before giving interpretations of our results, we need to justify the part of condition (10) which concerns the CES coefficients at the social level: this inequality ensures the existence of the bounds $\bar{\rho}_{0}$ and $\underline{\rho}_{0}$, and the possible occurrence of local indeterminacy when both elasticities of capital labor substitution are close to zero.

All the various conditions for the existence of indeterminacy in Proposition 4 are based on the restriction $\rho_{1}>\rho_{0}$ which implies a lower elasticity of capital-labor substitution in the investment good sector than in the consumption good sector. Since under Assumption 1 the elasticity of capital-labor substitution in the investment good sector is necessarily finite, it clearly appears that the same condition has to be considered for the investment good sector. In cases i), if the elasticity of capital-labor substitution in the consumption good sector is large enough, the extreme configuration of a Leontief technology in the investment good sector is also ruled out. On the contrary, if the factor substitutability of the consumption good sector is low enough, local indeterminacy becomes compatible with a Leontief technology in the investment good sector.

Let us now consider the configuration with $\beta_{10} \beta_{01} / \beta_{11} \beta_{00}>1$ and $\hat{\beta}_{10} \hat{\beta}_{01} / \hat{\beta}_{11} \hat{\beta}_{00}<1$ which is known to imply the existence of local indeterminacy in the case with Cobb-Douglas technologies in both sectors. ${ }^{3}$ Notice that Assumption 2 is then necessarily satisfied.

Proposition 5. Under Assumption 1, let

$$
\frac{\beta_{10} \beta_{01}}{\beta_{11} \beta_{00}}>1 \text { and } 1>\frac{\hat{\beta}_{10} \hat{\beta}_{01}}{\hat{\beta}_{11} \hat{\beta}_{00}}>\frac{\hat{\beta}_{01}}{\frac{\beta_{11}}{\delta+g}-\hat{\beta}_{11}}
$$

Then there are $\underline{\rho}_{0} \in(-1,0)$ and $\bar{\rho}_{0}>0$ such that the following results hold:

i) for any given $\rho_{0} \in\left(\underline{\rho}_{0}, \bar{\rho}_{0}\right)$, there exist $\underline{\rho}_{1} \in\left(\hat{\rho}_{1}, 0\right)$ and $\bar{\rho}_{1}>0$ such that the steady state is locally indeterminate when $\rho_{1} \in\left(\underline{\rho}_{1}, \bar{\rho}_{1}\right)$;

ii) for any given $\rho_{0}>\bar{\rho}_{0}$, there exists $\underline{\rho}_{1} \in\left(0, \rho_{0}\right)$ such that the steady state is locally indeterminate when $\rho_{1}>\underline{\rho}_{1}$.

Proposition 5 covers the formulation with symmetric elasticities of capitallabor substitution across sectors previously analyzed in Benhabib and

\footnotetext{
${ }^{3}$ See Benhabib and Nishimura [2].
} 
Nishimura [2] with Cobb-Douglas technologies and Nishimura and Venditti [3] with CES technologies having symmetric elasticities of capital-labor substitution. The part of condition (11) which concerns the CES coefficients at the social level is introduced to ensure the possibility of local indeterminacy when the consumption good technology is Cobb-Douglas while the investment good technology is Leontief (see case ii)). This explains why such a condition does not occur under the assumption of symmetric elasticities. However, since the plausible values of $\delta$ and $g$ are close to zero, this condition does not imply a strong restriction on the CES coefficients.

Contrary to the previous Proposition, the existence of local indeterminacy does not require the elasticity of capital-labor substitution in the consumption good sector to be larger than the one in the investment good sector. The extreme configuration of an infinite factor substitutability in the consumption good sector is again ruled out. Moreover, as shown in case ii), the occurrence of local indeterminacy with factors complementarity in the investment good sector is not compatible with a Cobb-Douglas technology in the consumption good sector and requires an elasticity of capital-labor substitution significantly lower than 1 .

Let us finally consider the configuration with $\hat{\beta}_{10} \hat{\beta}_{01} / \hat{\beta}_{11} \hat{\beta}_{00}>1$ which is known in the case with symmetric elasticities of capital-labor substitution to be compatible with the existence of local indeterminacy only when the common elasticity is lower than one, or $\rho_{0}=\rho_{1}=\rho>0 .^{4}$ In the next Proposition, we show that in the asymmetric case, $\rho_{0}$ has to be positive but under Assumption 1, $\rho_{1}$ can be negative, equal to 1 or positive.

Proposition 6. Under Assumptions 1-2, let

$$
\frac{\beta_{10} \beta_{01}}{\beta_{11} \beta_{00}}>1 \text { and } \frac{\hat{\beta}_{10} \hat{\beta}_{01}}{\hat{\beta}_{11} \hat{\beta}_{00}}>1
$$

Then there are $\underline{\rho}_{0}>0$ and $\bar{\rho}_{0}>\underline{\rho}_{0}$ such that the following results hold:

i) for any given $\rho_{0} \in\left(\underline{\rho}_{0}, \bar{\rho}_{0}\right)$, there exist $\underline{\rho}_{1} \in\left(\hat{\rho}_{1}, \rho_{0}\right)$ and $\bar{\rho}_{1}>0$ such that the steady state is locally indeterminate when $\rho_{1} \in\left(\underline{\rho}_{1}, \bar{\rho}_{1}\right)$;

\footnotetext{
${ }^{4}$ See Nishimura and Venditti [3].
} 
ii) for any given $\rho_{0}>\bar{\rho}_{0}$, there exists $\underline{\rho}_{1}>0$ such that the steady state is locally indeterminate when $\rho_{1}>\underline{\rho}_{1}$.

Proposition 6 confirms only part of the conclusions obtained by Nishimura and Venditti [3] under symmetric elasticities of capital-labor substitution across sectors: local indeterminacy requires some elasticity lower than one but only in the consumption good sector. Indeed, the existence of multiple equilibria does not rely on particular restrictions for the factor substitutability in the investment good sector. In particular, some elasticities greater than 1 are compatible with local indeterminacy in case i). However, as in the previous Propositions, a Leontief technology for the investment good can be reached only if the elasticity in the consumption good sector is low enough.

\section{Concluding comments}

Within the framework of CES technologies, we precisely show how much the elasticities of capital-labor substitution can differ across the industries while leading to local indeterminacy of equilibria. For instance, given a value for the elasticity of substitution in the consumption good sector, the elasticity of substitution in the investment good sector can be arbitrarily large.

\section{Appendix}

\subsection{Proof of Lemma 1}

Substituting (4) into the social production functions (2) gives

$$
y_{j}=\left(\hat{\beta}_{0 j} y_{j}^{-\rho_{j}}\left(\frac{p_{j} \beta_{0 j}}{w_{0}}\right)^{\frac{-\rho_{j}}{1+\rho_{j}}}+\hat{\beta}_{1 j} y_{j}^{-\rho_{j}}\left(\frac{p_{j} \beta_{1 j}}{w_{1}}\right)^{\frac{-\rho_{j}}{1+\rho_{j}}}\right)^{-1 / \rho_{j}}
$$

It follows that

$$
p_{j}^{\frac{\rho_{j}}{1+\rho_{j}}}=\left(\frac{\hat{\beta}_{0 j}}{\beta_{0 j}}\left(\frac{\beta_{0 j}}{w_{0}}\right)^{\frac{1}{1+\rho_{j}}} w_{0}+\frac{\hat{\beta}_{1 j}}{\beta_{1 j}}\left(\frac{\beta_{1 j}}{w_{1}}\right)^{\frac{1}{1+\rho_{j}}} w_{1}\right)^{-1 / \rho_{j}}
$$

Multiplying both sides by $p_{j}^{1 /\left(1+\rho_{j}\right)}$ then gives 


$$
p_{j}=\hat{a}_{0 j} w_{0}+\hat{a}_{1 j} w_{1}
$$

The result follows considering that $p_{0}=1$.

\subsection{Proof of Lemma 2}

By definition $x_{i j}=a_{i j} y_{j}$ and thus

$$
x_{i}=a_{i 0} y_{0}+a_{i 1} y_{1}
$$

The result follows considering that $x_{0}=1$.

\subsection{Proof of Lemma 3}

The result immediately follows from the fact that the function $\hat{A}(w, p)$ is homogeneous of degree zero in $w$ and $p$.

\subsection{Proof of Lemma 4}

The result follows from a direct differenciation of $x_{i}=a_{i 0} y_{0}+a_{i 1} y_{1}$ under the assumption that prices are constant.

\subsection{Proof of Proposition 1}

From equation (3) considered at the steady state with $y_{1}=g x_{1}$ and $w_{1}=$ $(\delta+g) p_{1}$, we get

$$
x_{11}=\left(\frac{\beta_{11}}{\delta+g}\right)^{\frac{1}{1+\rho_{1}}} g x_{1}
$$

Using now the social production function (2) for the investment good we derive

$$
x_{01}=\left(\frac{\left(\frac{\beta_{11}}{\delta+g}\right)^{\frac{\rho_{1}}{1+\rho_{1}}}-\hat{\beta}_{11}}{\hat{\beta}_{01}}\right)^{-\frac{1}{\rho_{1}}}\left(\frac{\beta_{11}}{\delta+g}\right)^{\frac{1}{1+\rho_{1}}} g x_{1}
$$

and thus

$$
\frac{x_{01}}{x_{11}}=\left(\frac{\left(\frac{\beta_{11}}{\delta+g}\right)^{\frac{\rho_{1}}{1+\rho_{1}}}-\hat{\beta}_{11}}{\hat{\beta}_{01}}\right)^{-\frac{1}{\rho_{1}}}
$$


Finally we easily obtain from (3):

$$
\begin{gathered}
\frac{\beta_{10} \beta_{01}}{\beta_{00} \beta_{11}}=\left(\frac{x_{01}}{x_{11}}\right)^{1+\rho_{1}}\left(\frac{x_{10}}{x_{00}}\right)^{1+\rho_{0}} \\
\Leftrightarrow \frac{x_{10}}{x_{00}}=\left(\frac{\beta_{10} \beta_{01}}{\beta_{00} \beta_{11}}\right)^{\frac{1}{1+\rho_{0}}}\left(\frac{\left(\frac{\beta_{11}}{\delta+g}\right)^{\frac{\rho_{1}}{1+\rho_{1}}}-\hat{\beta}_{11}}{\hat{\beta}_{01}}\right)^{\frac{1+\rho_{1}}{\rho_{1}\left(1+\rho_{0}\right)}}
\end{gathered}
$$

Considering (13), (14) and the fact that $x_{00}+x_{01}=1, x_{1}=x_{10}+x_{11}$, we get the final expression of $x_{1}^{*}$. Equation (3) for $i=1$ and $j=0$ gives

$$
w_{1}=\beta_{10}\left(\hat{\beta}_{11}\left(\frac{x_{10}}{x_{00}}\right)^{\rho_{0}}+\hat{\beta}_{10}\right)^{-\frac{1+\rho_{0}}{\rho_{0}}}
$$

The final expression of $p_{1}^{*}$ is then derived from (14) and the fact that $w_{1}=$ $(\delta+g) p_{1}$.

\subsection{Proof of Proposition 2}

i) From (4) we derive

$$
a_{11} a_{00}-a_{10} a_{01}=a_{00} a_{01}\left(\frac{a_{11}}{a_{01}}-\frac{a_{10}}{a_{00}}\right)=a_{00} a_{01} \frac{x_{11}}{x_{01}}\left(1-\frac{x_{10} x_{01}}{x_{00} x_{11}}\right)
$$

and the result follows after substitution of (13) and (14) into the previous expression.

ii) From (6) we derive

$$
\begin{aligned}
\hat{a}_{11} \hat{a}_{00}-\hat{a}_{10} \hat{a}_{01} & =\hat{a}_{00} \hat{a}_{01}\left(\frac{\hat{\beta}_{11}}{\hat{\beta}_{01}} \frac{\beta_{01}}{\beta_{11}} \frac{a_{11}}{a_{01}}-\frac{\hat{\beta}_{10}}{\hat{\beta}_{00}} \frac{\beta_{00}}{\beta_{10}} \frac{a_{10}}{a_{00}}\right) \\
& =a_{00} a_{01} \frac{\hat{\beta}_{11}}{\hat{\beta}_{01}} \frac{\beta_{01}}{\beta_{11}} \frac{x_{11}}{x_{01}}\left(1-\frac{\hat{\beta}_{10} \hat{\beta}_{01}}{\hat{\beta}_{00} \hat{\beta}_{11}} \frac{\beta_{00} \beta_{11}}{\beta_{10} \beta_{01}} \frac{x_{10} x_{01}}{x_{00} x_{11}}\right)
\end{aligned}
$$

and the result follows after substitution of (13) and (14) into the previous expression.

iii) The result is derived as in i) but considering instead the input coefficients at the social level (5).

\subsection{Proof of Proposition 3}

From Lemma 2, $x_{1}=a_{10} y_{0}+a_{11} y_{1}$. Moreover, at the steady state, $y_{1}=g x_{1}$, and it follows 


$$
a_{10} y_{0}+g a_{11} x_{1}=x_{1} \quad \Leftrightarrow \quad a_{10} y_{0}=\left[1-g a_{11}\right] x_{1}>0
$$

Therefore

$$
\frac{\partial y_{1}}{\partial x_{1}}-g=\frac{a_{00}}{a_{11} a_{00}-a_{10} a_{01}}-g=\frac{a_{00}\left[1-g a_{11}\right]+a_{10} a_{01} g}{a_{11} a_{00}-a_{10} a_{01}}<0
$$

if and only if $a_{11} a_{00}-a_{10} a_{01}<0$. From Lemma $1, p_{1}=\hat{a}_{01} w_{0}+\hat{a}_{11} w_{1}$. Moreover, at the steady state, $(\delta+g) p_{1}=w_{1}$, and it follows

$(\delta+g) \hat{a}_{01} w_{0}+(\delta+g) \hat{a}_{11} w_{1}=w_{1} \Leftrightarrow(\delta+g) \hat{a}_{01} w_{0}=\left[1-(\delta+g) \hat{a}_{11}\right] w_{1}>0$

Therefore

$$
\begin{aligned}
\delta+g-\frac{\partial w_{1}}{\partial p_{1}} & =\delta+g-\frac{\hat{a}_{00}}{\hat{a}_{11} \hat{a}_{00}-\hat{a}_{10} \hat{a}_{01}} \\
& =-\frac{\left[1-(\delta+g) \hat{a}_{11}\right] \hat{a}_{00}+(\delta+g) \hat{a}_{10} \hat{a}_{01}}{\hat{a}_{11} \hat{a}_{00}-\hat{a}_{10} \hat{a}_{01}}<0
\end{aligned}
$$

if and only if $\hat{a}_{11} \hat{a}_{00}-\hat{a}_{10} \hat{a}_{01}>0$.

\subsection{Proof of Proposition 4}

Proposition 3 shows that local indeterminacy occurs if and only if $b<0$ and $\hat{b}>0$. Under Assumption $1, \beta_{11}>\delta+g$ and $\rho_{1} \in\left(\hat{\rho}_{1},+\infty\right)$, while $\rho_{0}>-1$ without particular restriction. We will then study the sign of $b$ and $\hat{b}$ for different values of $\rho_{0}$ and $\rho_{1}$ over these intervals. Our strategy consists in considering a fixed value for $\rho_{0}$ and varying $\rho_{1}$ in order to find intervals of values in which local indeterminacy occurs. To simplify the analysis, we have to impose some restrictions on the parameters $\rho_{0}$ and $\rho_{1}$ such that the following function

$$
g\left(\rho_{1}\right)=\left(\frac{\left(\frac{\beta_{11}}{\delta+g}\right)^{\frac{\rho_{1}}{1+\rho_{1}}}-\hat{\beta}_{11}}{\hat{\beta}_{01}}\right)^{\frac{\rho_{1}-\rho_{0}}{\rho_{1}\left(1+\rho_{0}\right)}}
$$

is monotone increasing.

Lemma 5. Under Assumption 1, there is a $\tilde{\rho}_{0} \in(-1,0)$ such that for any given $\rho_{0}>\tilde{\rho}_{0}$ there exists $\tilde{\rho}_{1} \in\left[\hat{\rho}_{1}, 0\right)$ such that $g\left(\rho_{1}\right)$ is a monotone increasing function for all $\rho_{1}>\tilde{\rho}_{1}$.

Proof: Notice first that

$$
\frac{\partial}{\partial \rho_{1}}\left(\frac{\rho_{1}}{1+\rho_{1}}\right)=\frac{1}{\left(1+\rho_{1}\right)^{2}}>0, \quad \frac{\partial}{\partial \rho_{1}}\left(\frac{\rho_{1}-\rho_{0}}{\rho_{1}\left(1+\rho_{0}\right)}\right)=\frac{\rho_{0}}{\rho_{1}^{2}\left(1+\rho_{0}\right)}
$$


It follows that for any given $\rho_{0} \geq 0, g\left(\rho_{1}\right)$ is a monotone increasing function when $\rho_{1} \geq 0$. Consider now the case $\rho_{0} \in(-1,0)$. We can write $g\left(\rho_{1}\right)$ as follows:

$$
g\left(\rho_{1}\right)=\exp \left\{\frac{\rho_{1}-\rho_{0}}{\rho_{1}\left(1+\rho_{0}\right)} \ln \left(\frac{\exp \left[\frac{\rho_{1}}{1+\rho_{1}} \ln \left(\frac{\beta_{11}}{\delta+g}\right)\right]-\hat{\beta}_{11}}{\hat{\beta}_{01}}\right)\right\} \equiv \exp \left\{f\left(\rho_{1}\right)\right\}
$$

If $f\left(\rho_{1}\right)$ is monotone increasing, so in $g\left(\rho_{1}\right)$. We easily compute

$f^{\prime}\left(\rho_{1}\right)=\frac{\rho_{0}\left(1+\rho_{1}\right)^{2}\left[\left(\frac{\beta_{11}}{\delta+g}\right)^{\frac{\rho_{1}}{1+\rho_{1}}}-\hat{\beta}_{11}\right] \ln \left(\frac{\left(\frac{\beta_{11}}{\delta+g}\right)^{\frac{\rho_{1}}{1+\rho_{1}}}-\hat{\beta}_{11}}{\hat{\beta}_{01}}\right)+\rho_{1}\left(\rho_{1}-\rho_{0}\right)\left(\frac{\beta_{11}}{\delta+g}\right)^{\frac{\rho_{1}}{1+\rho_{1}}} \ln \left(\frac{\beta_{11}}{\delta+g}\right)}{\rho_{1}^{2}\left(1+\rho_{1}\right)^{2}\left(1+\rho_{0}\right)\left[\left(\frac{\beta_{11}}{\delta+g}\right)^{\frac{\rho_{1}}{1+\rho_{1}}}-\hat{\beta}_{11}\right]}$

We have now to compute the sign of $\lim _{\rho_{1} \rightarrow 0} f^{\prime}\left(\rho_{1}\right)$. A Taylor expansion of order two allows to show that $\lim _{\rho_{1} \rightarrow 0} f^{\prime}\left(\rho_{1}\right)>0$ for any $\rho_{0}>\tilde{\rho}_{0}$ with

$$
\tilde{\rho}_{0}=-\left[1+\ln \left(\frac{\beta_{11}}{\delta+g}\right) \frac{\hat{\beta}_{11}}{2 \hat{\beta}_{01}}\right]^{-1} \in\left(-1, \hat{\rho}_{1}\right)
$$

Notice finally that $\lim _{\rho_{1} \rightarrow \hat{\rho}_{1}} f^{\prime}\left(\rho_{1}\right)=-\infty$ and $\lim _{\rho_{1} \rightarrow \hat{\rho}_{1}} f\left(\rho_{1}\right)=+\infty$ if $\rho_{0}<$ $\hat{\rho}_{1}$, while $\lim _{\rho_{1} \rightarrow \hat{\rho}_{1}} f^{\prime}\left(\rho_{1}\right)=+\infty$ and $\lim _{\rho_{1} \rightarrow \hat{\rho}_{1}} f\left(\rho_{1}\right)=-\infty$ if $\rho_{0}>\hat{\rho}_{1}$. We then conclude from all this that for any given $\rho_{0}>\tilde{\rho}_{0}$, there exists $\tilde{\rho}_{1} \in\left[\hat{\rho}_{1}, 0\right)$ such that $g\left(\rho_{1}\right)$ is a monotone increasing function for all $\rho_{1}>\tilde{\rho}_{1}$.

It clearly appears from Proposition 2 that under the conditions of Lemma $5, b$ and $\hat{b}$ are monotone decreasing function of $\rho_{1}$.

We may now prove Proposition 4: In the first part of the proof we consider positive values of $\rho_{1}$ only. Notice that L'Hôpital's rule gives:

$$
\lim _{\rho_{1} \rightarrow 0} g\left(\rho_{1}\right)=\left(\frac{\beta_{11}}{\delta+g}\right)^{\frac{-\rho_{0}}{\left(1+\rho_{0}\right) \hat{\beta}_{01}}}
$$

Consider the capital intensity difference at the private level $b$. The previous result implies

$$
\lim _{\rho_{1} \rightarrow 0} b=1-\left(\frac{\beta_{10} \beta_{01}}{\beta_{00} \beta_{11}}\right)^{\frac{1}{1+\rho_{0}}}\left(\frac{\delta+g}{\beta_{11}}\right)^{\frac{\rho_{0}}{\left(1+\rho_{0}\right) \hat{\beta}_{01}}}
$$

Notice that the right-hand-side of (17) is a monotone function of $\rho_{0}$. Under Assumption 2 and $\beta_{10} \beta_{01} / \beta_{11} \beta_{00}<1$, we derive $\left.\lim _{\rho_{1} \rightarrow 0} b\right|_{\rho_{0} \geq 0}>0$. It follows that there exists $\rho_{0}^{1} \in[-1,0)$ such that $\lim _{\rho_{1} \rightarrow 0} b>0$ for any $\rho_{0}>\rho_{0}^{1}$. Similarly, we get 


$$
\lim _{\rho_{1} \rightarrow+\infty} b=1-\left(\frac{\beta_{10} \beta_{01}}{\beta_{00} \beta_{11}}\right)^{\frac{1}{1+\rho_{0}}}\left(\frac{\frac{\beta_{11}}{\delta+g}-\hat{\beta}_{11}}{\hat{\beta}_{01}}\right)^{\frac{1}{1+\rho_{0}}}
$$

Notice again that the right-hand-side of (18) is a monotone function of $\rho_{0}$. If the following condition holds

$$
\frac{\beta_{10} \beta_{01}}{\beta_{00} \beta_{11}} \frac{\frac{\beta_{11}}{\delta+g}-\hat{\beta}_{11}}{\hat{\beta}_{01}}>1
$$

then $\left.\lim _{\rho_{1} \rightarrow+\infty} b\right|_{\rho_{0} \rightarrow+\infty}=0$ - and $\left.\lim _{\rho_{1} \rightarrow+\infty} b\right|_{\rho_{0} \leq 0}<0$. It follows that under (19), $\lim _{\rho_{1} \rightarrow+\infty} b<0$ for any $\rho_{0}>-1$. Notice finally that if $\rho_{0}=\rho_{1}$ we have $b>0$. Considering Lemma 5 under (19), we then conclude that for any given $\rho_{0}>\rho_{0}^{1}$, there exists $\rho_{1}^{1}>\max \left\{0, \rho_{0}\right\}$ such that $b<0$ for all $\rho_{1}>\rho_{1}^{1}$.

Consider now the quasi capital intensity difference at the social level $\hat{b}$. Equation (16) implies

$$
\lim _{\rho_{1} \rightarrow 0} \hat{b}=1-\frac{\frac{\hat{\beta}_{10} \hat{\beta}_{01}}{\hat{\beta}_{11} \hat{\beta}_{00}}}{\frac{\beta_{10} \beta_{01}}{\beta_{11} \beta_{00}}}\left(\frac{\beta_{10} \beta_{01}}{\beta_{00} \beta_{11}}\right)^{\frac{1}{1+\rho_{0}}}\left(\frac{\delta+g}{\beta_{11}}\right)^{\frac{\rho_{0}}{\left(1+\rho_{0}\right) \hat{\beta}_{01}}}
$$

Notice that the right-hand-side of (20) is a monotone function of $\rho_{0}$. Under Assumption 2 and $\hat{\beta}_{10} \hat{\beta}_{01} / \hat{\beta}_{11} \hat{\beta}_{00}<1$, we derive $\left.\lim _{\rho_{1} \rightarrow 0} \hat{b}\right|_{\rho_{0} \geq 0}>0$ so that there exists $\rho_{0}^{2} \in[-1,0)$ such that $\lim _{\rho_{1} \rightarrow 0} \hat{b}>0$ for any $\rho_{0}>\rho_{0}^{2}$. Since $\hat{b}>b$, we get when $\rho_{0}=\rho_{0}^{1},\left.b\right|_{\rho_{1}=0}=0$ while $\left.\hat{b}\right|_{\rho_{1}=0}>0$ so that $\rho_{0}^{2}<\rho_{0}^{1}$. Similarly, we have

$$
\lim _{\rho_{1} \rightarrow+\infty} \hat{b}=1-\frac{\frac{\hat{\beta}_{10} \hat{\beta}_{01}}{\hat{\beta}_{11} \hat{\beta}_{00}}}{\frac{\beta_{10} \beta_{01}}{\beta_{11} \beta_{00}}}\left(\frac{\beta_{10} \beta_{01}}{\beta_{00} \beta_{11}}\right)^{\frac{1}{1+\rho_{0}}}\left(\frac{\frac{\beta_{11}}{\delta+g}-\hat{\beta}_{11}}{\hat{\beta}_{01}}\right)^{\frac{1}{1+\rho_{0}}}
$$

Notice again that the right-hand-side of (21) is a monotone function of $\rho_{0}$. If the following condition holds

$$
\frac{\hat{\beta}_{10} \hat{\beta}_{01}}{\hat{\beta}_{11} \hat{\beta}_{00}} \frac{\frac{\beta_{11}}{\delta+g}-\hat{\beta}_{11}}{\hat{\beta}_{01}}>1
$$

we get with $\left.\lim _{\rho_{1} \rightarrow+\infty} \hat{b}\right|_{\rho_{0}=0}<0$ and $\left.\lim _{\rho_{1} \rightarrow+\infty} \hat{b}\right|_{\rho_{0} \rightarrow+\infty}>0$. Therefore, under (22), there exists $\rho_{0}^{3}>0$ such that $\lim _{\rho_{1} \rightarrow+\infty} \hat{b}>0$ for any $\rho_{0}>\rho_{0}^{3}$. Considering Lemma 5 under (22), we then conclude the following results:

- for any given $\rho_{0} \in\left(\rho_{0}^{2}, \rho_{0}^{3}\right)$, there exists $\rho_{1}^{2}>0$ such that $\hat{b}>0$ for all $\rho_{1} \in\left[0, \rho_{1}^{2}\right)$. Notice that since $\hat{b}>b, \rho_{1}^{2}>\rho_{1}^{1}$;

- for any given $\rho_{0}>\rho_{0}^{3}, \hat{b}>0$ for all $\rho_{1}>\rho_{0}$. 
Notice that under Assumption 2, (22) implies (19). Therefore, under (22), we conclude that local indeterminacy occurs in the following cases:

i) for any given $\rho_{0} \in\left(\max \left\{\rho_{0}^{1}, \rho_{0}^{2}\right\}, \rho_{0}^{3}\right)$ and $\rho_{1} \in\left(\underline{\rho}_{1}, \bar{\rho}_{1}\right)$ with $\underline{\rho}_{1}=\rho_{1}^{1}>$ $\max \left\{0, \rho_{0}\right\}$ and $\bar{\rho}_{1}=\rho_{1}^{2}>\underline{\rho}_{1}$;

ii) for any given $\rho_{0}>\rho_{0}^{3}$ and $\rho_{1}>\underline{\rho}_{1}$ with $\underline{\rho}_{1}=\rho_{1}^{2}>\rho_{0}$.

\subsection{Proof of Proposition 5}

We use the same kind of arguments as in Proposition 4. Consider first the capital intensity difference at the private level $b$. Under Assumption 2 and $\beta_{10} \beta_{01} / \beta_{11} \beta_{00}>1$, we derive from (17) that $\left.\lim _{\rho_{1} \rightarrow 0} b\right|_{\rho_{0} \leq 0}<0$ while $\left.\lim _{\rho_{1} \rightarrow 0} b\right|_{\rho \rightarrow+\infty}>0$. Therefore, there exists $\rho_{0}^{1}>0$ such that $\lim _{\rho_{1} \rightarrow 0} b<0$ for any $\rho_{0} \in\left(-1, \rho_{0}^{1}\right)$. Similarly, we derive from (18) that $\lim _{\rho_{1} \rightarrow+\infty} b<0$ for any $\rho_{0}>-1$. Notice also that

$$
\left.b\right|_{\rho_{1}=\rho_{0}}=1-\left(\frac{\beta_{10} \beta_{01}}{\beta_{00} \beta_{11}}\right)^{\frac{1}{1+\rho_{0}}}<0
$$

Considering Lemma 5, we then conclude the following results:

a) for any given $\rho_{0} \in\left(-1, \rho_{0}^{1}\right), b<0$ for all $\rho_{1} \geq 0$;

b) for any given $\rho_{0}>\rho_{0}^{1}$, there exists $\rho_{1}^{1} \in\left(0, \rho_{0}\right)$ such that $b<0$ for all $\rho_{1}>\rho_{1}^{1}$.

Consider now the quasi capital intensity difference at the social level $\hat{b}$. Under Assumption 2 and $\hat{\beta}_{10} \hat{\beta}_{01} / \hat{\beta}_{11} \hat{\beta}_{00}<1$, we derive from $(20)$ that $\left.\lim _{\rho_{1} \rightarrow 0} \hat{b}\right|_{\rho_{0} \geq 0}>0$ while $\left.\lim _{\rho_{1} \rightarrow 0} \hat{b}\right|_{\rho_{0} \rightarrow-1}<0$. Therefore, there exists $\rho_{0}^{2} \in$ $(-1,0)$ such that $\lim _{\rho_{1} \rightarrow 0} \hat{b}>0$ for any $\rho_{0}>\rho_{0}^{2}$. Similarly, we derive from (21) that $\left.\lim _{\rho_{1} \rightarrow+\infty} \hat{b}\right|_{\rho_{0}=0}<0$ if (22) holds, while $\left.\lim _{\rho_{1} \rightarrow+\infty} \hat{b}\right|_{\rho_{0} \rightarrow+\infty}>0$. Therefore, under (22), there exists $\rho_{0}^{3}>0$ such that $\lim _{\rho_{1} \rightarrow+\infty} \hat{b}>0$ for any $\rho_{0}>\rho_{0}^{3}$. Notice also that

$$
\left.\hat{b}\right|_{\rho_{1}=\rho_{0}}=1-\frac{\hat{\beta}_{10} \hat{\beta}_{01}}{\hat{\beta}_{11} \hat{\beta}_{00}}\left(\frac{\beta_{00} \beta_{11}}{\beta_{10} \beta_{01}}\right)^{\frac{\rho_{0}}{1+\rho_{0}}}
$$

is an increasing function of $\rho_{0}$ since $\beta_{00} \beta_{11} / \beta_{10} \beta_{01}<1$. Considering that $\rho_{0}^{2} \in(-1,0)$ is defined as $\left.\lim _{\rho_{1} \rightarrow 0} \hat{b}\right|_{\rho_{0}=\rho_{0}^{2}}=0$, we derive from $(20)$ :

$$
\left.\hat{b}\right|_{\rho_{1}=\rho_{0}=\rho_{0}^{2}}=1-\left(\frac{\beta_{11}}{\delta+g}\right)^{\frac{\rho_{0}^{2}}{\left(1+\rho_{0}^{2}\right) \hat{\beta}_{01}}}>0
$$


Therefore $\left.\hat{b}\right|_{\rho_{1}=\rho_{0}}>0$ for any $\rho_{0} \geq \rho_{0}^{2}$.

Considering Lemma 5 under (22), we then conclude the following results:

c) for any given $\rho_{0} \in\left(\rho_{0}^{2}, \rho_{0}^{3}\right)$, there exists $\rho_{1}^{2}>\max \left\{0, \rho_{0}\right\}$ such that $\hat{b}>0$ for all $\rho_{1} \in\left[0, \rho_{1}^{2}\right)$;

d) for any given $\rho_{0}>\rho_{0}^{3}, \hat{b}>0$ for all $\rho_{1} \geq 0$.

Therefore, under (22), we conclude from points a)-e) that local indeterminacy occurs in the following cases:

i) for any given $\rho_{0} \in\left(\rho_{0}^{2}, \max \left\{\rho_{0}^{1}, \rho_{0}^{3}\right\}\right)$ and $\rho_{1} \in\left(\underline{\rho}_{1}, \bar{\rho}_{1}\right)$ with $\underline{\rho}_{1} \in\left(\hat{\rho}_{1}, 0\right)$ and $\bar{\rho}_{1}=\rho_{1}^{2}>\max \left\{0, \rho_{0}\right\}$;

ii) for any given $\rho_{0}>\max \left\{\rho_{0}^{1}, \rho_{0}^{3}\right\}$ and $\rho_{1}>\underline{\rho}_{1}$ with $\underline{\rho}_{1}=\rho_{1}^{1}>0$.

\subsection{Proof of Proposition 6}

We use the same kind of arguments as in Proposition 5. Consider first the capital intensity difference at the private level $b$. Under Assumption 2 and $\beta_{10} \beta_{01} / \beta_{11} \beta_{00}>1$, we have already shown in the proof of Proposition 5 that there exists $\rho_{0}^{1}>0$ such that:

a) for any given $\rho_{0} \in\left(-1, \rho_{0}^{1}\right), b<0$ for all $\rho_{1} \geq 0$;

b) for any given $\rho_{0}>\rho_{0}^{1}$, there exists $\rho_{1}^{1} \in\left(0, \rho_{0}\right)$ such that $b<0$ for all $\rho_{1}>\rho_{1}^{1}$.

Consider now the quasi capital intensity difference at the social level $\hat{b}$. Under Assumption 2 and $\hat{\beta}_{10} \hat{\beta}_{01} / \hat{\beta}_{11} \hat{\beta}_{00}>1$, we derive from $(20)$ that $\left.\lim _{\rho_{1} \rightarrow 0} \hat{b}\right|_{\rho_{0}=0}<0$ while $\left.\lim _{\rho_{1} \rightarrow 0} \hat{b}\right|_{\rho_{0} \rightarrow+\infty}>0$. Therefore, there exists $\rho_{0}^{2}>0$ such that $\lim _{\rho_{1} \rightarrow 0} \hat{b}>0$ for any $\rho_{0}>\rho_{0}^{2}$. Since $\hat{b}>b$, we get $\rho_{0}^{2}<\rho_{0}^{1}$. Similarly, we derive from $(21)$ that $\left.\lim _{\rho_{1} \rightarrow+\infty} \hat{b}\right|_{\rho_{0}=0}<0$ while $\left.\lim _{\rho_{1} \rightarrow+\infty} \hat{b}\right|_{\rho_{0} \rightarrow+\infty}>0$. Therefore, there exists $\rho_{0}^{3}>0$ such that $\lim _{\rho_{1} \rightarrow+\infty} \hat{b}>0$ for any $\rho_{0}>\rho_{0}^{3}$.

Now notice from (20) and (21) the following property:

$$
\left.\lim _{\rho_{1} \rightarrow+\infty} \hat{b}\right|_{\rho_{0}=\rho_{0}^{2}}=1-\left(\frac{\frac{\beta_{11}}{\delta+g}-\hat{\beta}_{11}}{\hat{\beta}_{01}}\right)^{\frac{1}{1+\rho_{0}^{2}}}\left(\frac{\beta_{11}}{\delta+g}\right)^{\frac{\rho_{0}^{2}}{\left(1+\rho_{0}^{2}\right) \hat{\beta}_{01}}}<0
$$

Therefore we get $\rho_{0}^{2}<\rho_{0}^{3}$.

Consider finally equations $(24)$ and $(26)$. Since $\rho_{0}^{2}>0$ is such that $\left.\lim _{\rho_{1} \rightarrow 0} \hat{b}\right|_{\rho_{0}=\rho_{0}^{2}}=0$, we derive from $(20):\left.\hat{b}\right|_{\rho_{1}=\rho_{0}=\rho_{0}^{2}}<0$. On the con- 
trary, since $\rho_{0}^{3}>0$ is such that $\left.\lim _{\rho_{1} \rightarrow+\infty} \hat{b}\right|_{\rho_{0}=\rho_{0}^{3}}=0$, we derive from $(21)$ :

$$
\left.\hat{b}\right|_{\rho_{1}=\rho_{0}=\rho_{0}^{3}}=1-\left(\frac{\hat{\beta}_{01}}{\frac{\beta_{11}-\hat{\beta}_{11}}{\delta+g}}\right)^{\frac{1}{1+\rho_{0}^{3}}}>0
$$

Therefore we get $\left.\hat{b}\right|_{\rho_{1}=\rho_{0}}>0$ for any $\rho_{0} \geq \rho_{0}^{3}$.

Considering Lemma 5, we then conclude the following results:

c) for any given $\rho_{0} \in\left(\rho_{0}^{2}, \rho_{0}^{3}\right)$, there exists $\rho_{1}^{2} \in\left(0, \rho_{0}\right]$ such that $\hat{b}>0$ for all $\rho_{1} \in\left[0, \rho_{1}^{2}\right)$;

d) for any given $\rho_{0}>\rho_{0}^{3}, \hat{b}>0$ for all $\rho_{1} \geq 0$.

Notice that since $\hat{b}>b$, we get $\rho_{1}^{2}>\rho_{1}^{1}$.

Therefore, we conclude from points a)-d) that local indeterminacy occurs in the following cases:

i) for any given $\rho_{0} \in\left(\rho_{0}^{2}, \max \left\{\rho_{0}^{1}, \rho_{0}^{3}\right\}\right)$ and $\rho_{1} \in\left(\underline{\rho}_{1}, \bar{\rho}_{1}\right)$ with $\underline{\rho}_{1} \in\left(\hat{\rho}_{1}, \rho_{0}\right)$ and $\bar{\rho}_{1}=\rho_{1}^{2} \in\left(0, \rho_{0}\right]$;

ii) for any given $\rho_{0}>\max \left\{\rho_{0}^{1}, \rho_{0}^{3}\right\}$ and $\rho_{1}>\underline{\rho}_{1}$ with $\underline{\rho}_{1}=\rho_{1}^{1} \in\left(0, \rho_{0}\right)$.

\section{References}

[1] Benhabib, J. and R. Farmer (1999): "Indeterminacy and Sunspots in Macroeconomics," in Handbook of Macroeconomics, J.B. Taylor and M. Woodford (eds.), North-Holland, Amsterdam, 387-448.

[2] Benhabib, J., and K. Nishimura (1998): "Indeterminacy and Sunspots with Constant Returns," Journal of Economic Theory, 81, 58-96.

[3] Nishimura, K., and A. Venditti (2004): "Indeterminacy and the Role of Factor Substitutability," Macroeconomic Dynamics, 8, 436-465. 